Check for updates

Cite this: Chem. Commun., 2017, 53,4250

Received 8th February 2017 Accepted 27th March 2017

DOI: $10.1039 / c 7 c c 01023 k$

rsc.li/chemcomm

\section{Controlling transmembrane protein concentration and orientation in supported lipid bilayers $\uparrow$}

\author{
P. Bao, (D) ${ }^{a}$ M. L. Cartron, ${ }^{\text {b K. H. Sheikh, }}{ }^{c}$ B. R. G. Johnson, ${ }^{a}$ C. N. Hunter ${ }^{b}$ and \\ S. D. Evans ${ }^{\star a}$
}

The trans-membrane protein - proteorhodopsin ( $p R$ ) has been incorporated into supported lipid bilayers (SLB). In-plane electric fields have been used to manipulate the orientation and concentration of these proteins, within the SLB, through electrophoresis leading to a 25-fold increase concentration of $\mathrm{pR}$.

The cell membrane defines the boundary between the cell interior and the extracellular environment and acts as an impermeable barrier to the transfer of ions and small molecules and thus allows the generation of electrical and chemical gradients. Further, it acts as host to a wide variety of membrane proteins that regulate a range of cellular processes and represent the targets of more than $60 \%$ of modern pharmaceuticals. ${ }^{1}$ Membrane proteins are difficult to study because of their inherent instability outside of their membranous environment and this has led to a variety of approaches being investigated, including the use of lipid discs; droplet interface bilayers; and solid supported, or tethered, lipid bilayer (SLBs). ${ }^{2-6}$ The latter have potential for the study of membrane protein function, peptide-membrane interactions and for understanding the role of phase separation and domains in lipid membranes. ${ }^{7-10}$ Typically, SLBs are formed via vesicle adsorption, rupture and fusion at the surface. ${ }^{11}$ This process is effective for lipid-only vesicles and vesicles with low protein content. Numerous studies have demonstrated the incorporation of functional membrane proteins into such bilayers and those involved in the photosynthetic process have recently been reviewed. ${ }^{12}$ However, at higher protein concentrations vesicles tend to remain intact thereby limiting the amount of protein that can be incorporated within the SLB. ${ }^{13,14}$ It is therefore of interest to develop methods to increase membrane protein concentration, and orientation, post bilayer formation. ${ }^{5,15-22}$ Approaches followed

\footnotetext{
${ }^{a}$ School of Physics \& Astronomy, University of Leeds, LS2 9JT, UK.

E-mail: s.d.evans@leeds.ac.uk

${ }^{b}$ Department of Molecular Biology \& Biotechnology, University of Sheffield,

S10 2TH, UK

${ }^{c}$ School of Biomedical Science, University of Leeds, LS2 9JT, UK

$\dagger$ Electronic supplementary information (ESI) available: FRAP, AFM results for

bilayer on glass and mica, and experiment details. See DOI: 10.1039/c7cc01023k
}

to date include the use of hydrodynamic drag, which relies on the frictional interaction between a fluid flowing across the membrane and protruding membrane components. ${ }^{15,23}$ Jönsson et al., have used this to demonstrate the accumulation and separation of membrane associated proteins, streptavidin and cholera toxin, coupled to receptors in lipid membrane. ${ }^{15}$ Alternatively, electric fields can be used to induce electrophoretic and electro-osmotic manipulation of charged species. ${ }^{13,19,24-27}$ Pace et al. recently demonstrated the successful separation of four membraneassociated species according to their different size/charge ratio. ${ }^{19}$ Further, we have shown the possibility of moving and concentrating an integral membrane protein, Cyma, which has a single membrane spanning $\alpha$-helix. ${ }^{17}$ However, until now, there have been no reports on the electric-field based manipulation of larger integral membrane proteins within SLBs.

The light-activated proton pump, proteorhodopsin ( $\mathrm{pR})$, has seven membrane spanning domains and charged hydrophilic loops extending beyond the membrane with a molecular weight of $\sim 29 \mathrm{kDa}$. It can be expressed in $E$. coli and has broad potential application from photovoltaic to data storage. ${ }^{28-30}$ Here we show that pRs reconstituted into lipid bilayers are mobile and can be concentrated via the application of in-plane electric field (Fig. 1).

SLBs were formed from $\sim 50 \mathrm{~nm}$ diameter vesicles of DOPC, containing $1 \mathrm{wt} \%$ Alexa488 labelled $\mathrm{pR} .{ }^{31}$ Bilayer formation was evaluated using fluorescence microscopy and AFM. Fluorescence recovery after photo-bleaching (FRAP) data for Alexa488 labelled protein (pR-Alexa488) shows that the $\mathrm{pR}$ is mobile with a diffusion coefficient $(D)$ of $0.39 \pm 0.03 \mu \mathrm{m}^{2} \mathrm{~s}^{-1}$ and the mobile fraction $87 \pm 5 \%$ (Fig. $\mathrm{S} 1, \mathrm{ESI} \dagger$ ). This value for $D$ is lower than the 1 to $4 \mu \mathrm{m}^{2} \mathrm{~s}^{-1}$ typically observed for lipids in SLBs formed on glass but similar to observations for other membrane proteins in SLBs. $^{32,33}$ For example haemolysin $\left(M_{\mathrm{w}}=33 \mathrm{kDa}\right)$ has been observed diffuse 3-fold slower than lipids in the SLBs on glass. ${ }^{33}$ Since the $\mathrm{pR}$ has an extra-membranous region that extends beyond the surface of bilayer by nearly $1 \mathrm{~nm}$ interaction with the underlying glass substrate could also contribute to reduced diffusivity and mobile fraction values. ${ }^{34-36}$ 


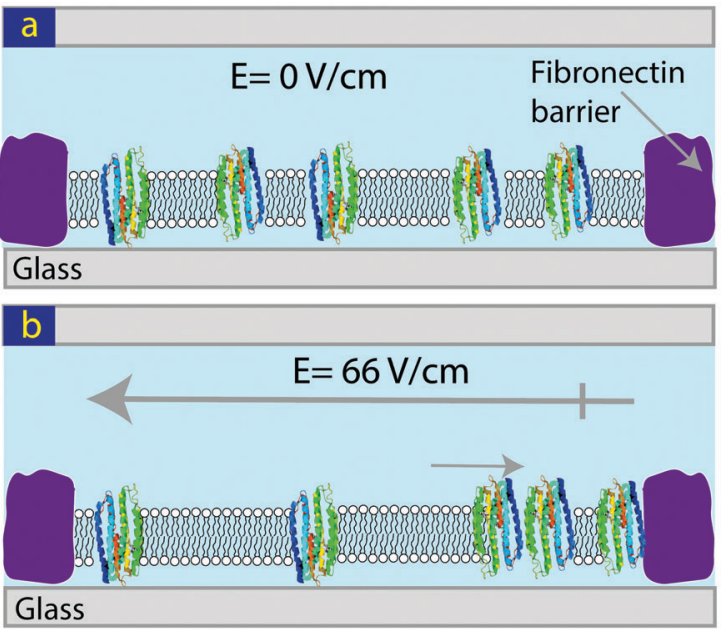

Fig. 1 Schematic showing the concentration of $\mathrm{pR}$ in a SLB using an in-plane electric field. (a) Before, and (b) following the application of an E-field.

The force-distance curve obtained by AFM of pR-containing lipid bilayers on glass (Fig. 2a) show typical a "punch-through" profile found for SLBs and indicates a bilayer thickness of $\sim 5.6 \pm 0.2 \mathrm{~nm}(n=7) \cdot{ }^{37}$ A particle density of $\sim 12 \pm 9$ per $\mu \mathrm{m}^{2}$ $(n=19)$, was observed when imaging SLBs on glass (Fig. 2b). Since the AFM scan speeds were only up to $10 \mu \mathrm{m} \mathrm{s}^{-1}$ this does not allow the observation of the mobile fraction of proteins and these particles are likely to be immobile pRs oligomers, as shown in Fig. S4c (ESI $\dagger$ ). Control experiments on pure DOPC lipid bilayers on glass didn’t show any particles, Fig. S3 (ESI†). However, they did show the presence of some nanosized holes $(25 \pm 3 \mathrm{~nm}$ in diameter, $n=20)$ in both the case of pR-containing,
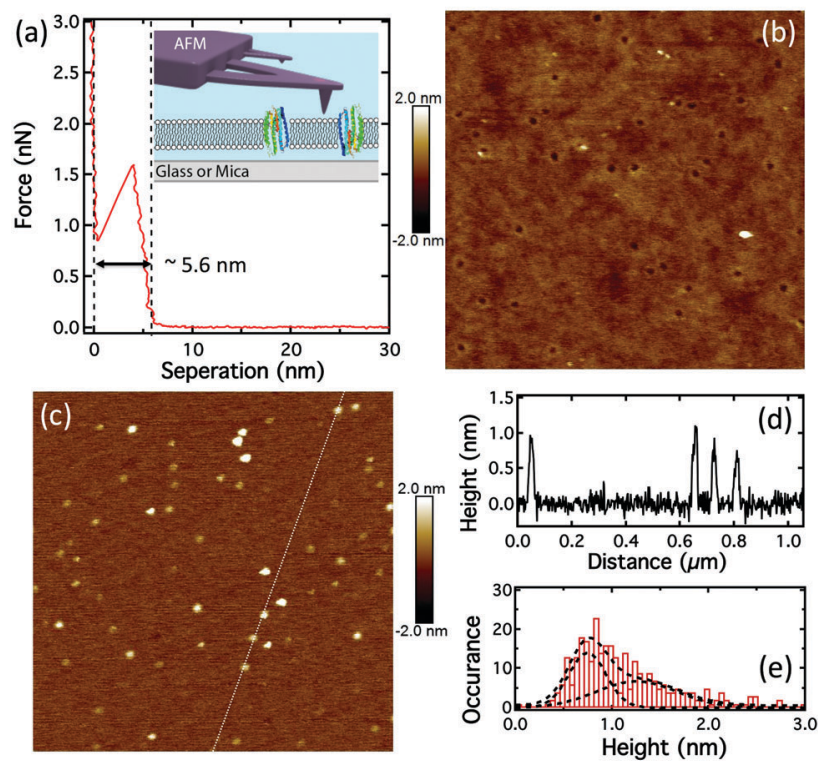

Fig. 2 pR-Containing SLB formation. (a) The force-separation curve showing "punch-through" to determine bilayer thickness; inset: schematic of the AFM on a SLB. (b) AFM height image of pRs in SLB on glass (1 $\mu \mathrm{m}$ scan). (c) AFM height image of pRs in SLB on mica (1 $\mu \mathrm{m}$ scan). (d) The height profile along the dotted line in (c). (e) The histogram of the pR height, on mica, with fits to two peaks located at 0.8 and $1.3 \mathrm{~nm}$ respectively. and pure, lipid bilayers on glass (Fig. $2 \mathrm{~b}$ and Fig. S2, S3, ESI $\dagger$ ). These holes are not found in bilayers formed on mica (Fig. S4 and S5, ESI $\dagger$ ). AFM imaging of the glass coverslips (no. 1 Menzel Co., Germany) indicated that these were in fact due to the defects in glass substrate (Fig. S3, ESI†). AFM imaging of pRs in the SLB on mica showed a greater proportion of immobile particles than that on glass (Fig. 2c and Fig. S4, ESI $\dagger$ ), presumably due to increased interaction with the mica. The pRs protrude from the bilayer by around $1 \mathrm{~nm}$ (Fig. 2d). From fitting the height data we observe two populations of pRs that protrude on average by $\sim 0.8$ and $1.3 \mathrm{~nm}$ (Fig. 2e). These values are close to the $0.9 \mathrm{~nm}$ reported by Klyszejko et al. for pR crystals. ${ }^{38}$ The different the heights likely reflect the two possible orientations of the pRs in SLBs and is supported by the similar percentages of the two populations $(49 \%$ and $51 \%$, respectively), which is expected from a nearly random formation of SLBs via vesicle rupture. Single particle tracking (SPT) of these proteins in SLBs on mica suggested pRs are immobile (Fig. S6, ESI $†$ ), therefore, the density of pRs on mica should roughly represent the density of pRs in the proteoliposome, as well as in the bilayer on glass. Particle analysis suggests the density of pR in the lipid bilayer is $50 \pm 10 \mu^{-2}(n=10)$. From the initial concentration we would expect to observe $\sim 350 \mathrm{pR}$ molecules per $\mu \mathrm{m}^{2}$ suggesting that the particles observed consist of oligomers of protein (predominantly hexamers). This is consistent with the earlier report of oligomer states of $\mathrm{pR}$ in the membrane of E. coli. ${ }^{39}$

To demonstrate the possibility of controlling the concentration of transmembrane proteins, bilayers containing $1 \mathrm{wt} \%$ pR-Alexa 488 were formed by vesicle fusion of $50 \mathrm{~nm}$ proteoliposomes onto non-fibronectin covered regions of the surface. ${ }^{17}$ The fluorescence images of the patterned lipid bilayer for pR-Alexa488 are shown in Fig. 3. During the application of an electric field, $66 \mathrm{~V} \mathrm{~cm}^{-1}$, the pR-Alexa488 within the SLB moved toward the positive electrode and built-up as a function of time, as shown in Fig. 3a and b. These show that most of pR-Alexa488 molecules are concentrated in the trap regions. By increasing the length, $L$, of the bilayer structures we are able to systematically control the concentration of $\mathrm{pR}$. The average fluorescence intensity for pR-Alexa488 in the trap head region (defined by white dotted line) for the longest device showed a $\sim 7$-fold increase in intensity after application of the E-field. This value is lower than the maximum possible value of ' $\times 18$ ' estimated from the area ratio between the whole trap and the head region for corral 6 and is possibly due to lower pR mobility in increasingly crowded local environments, as indicated by the accumulation curves shown in Fig. 3c.

The build-up of pR was followed by AFM of the bilayer after electrophoresis, Fig. 4a. The density of pR in the head of the longest trap (labeled by an asterisk in the corral 6 in Fig. 3b) was $\sim 1264 \pm 76 \mu^{-2}$ after electrophoresis (Fig. 4a) compared to a density of $\sim 50 \mu \mathrm{m}^{-2}$ prior to the application of the field (Fig. 2c) indicating a 25 fold build-up of pR concentration. We note that comparison between AFM and fluorescence is not straight forward as the AFM measures a local concentration within the trap and we expect an exponential profile in the protein distribution against the end of the barrier. Notwithstanding this, the AFM indicates that a significantly increased concentration of pRs 

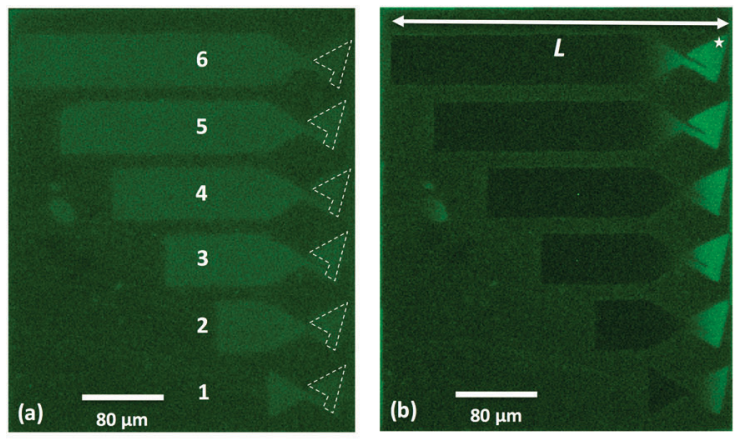

(c)

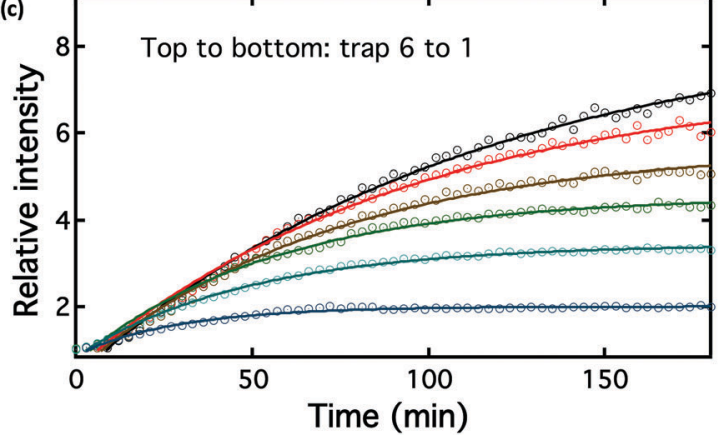

Fig. 3 Electrophoresis of pR-Alexa488 in the DOPC lipid bilayer (brighter color regions) in "trap" structures of different lengths (labeled 1-6) formed by microcontact-printing fibronectin (darker color region) on a glass substrate. False-color fluorescence images of pR-Alexa488 using a FITC filter: (a) before application of E-field; (b) after E-field applied for180 min; (c) accumulation curves of pR-Alexa488 in the trap heads (regions defined by dashed lines in (a)) as a function of time. The star in (b) indicates where the AFM images were taken following the application of E-field.

(a)

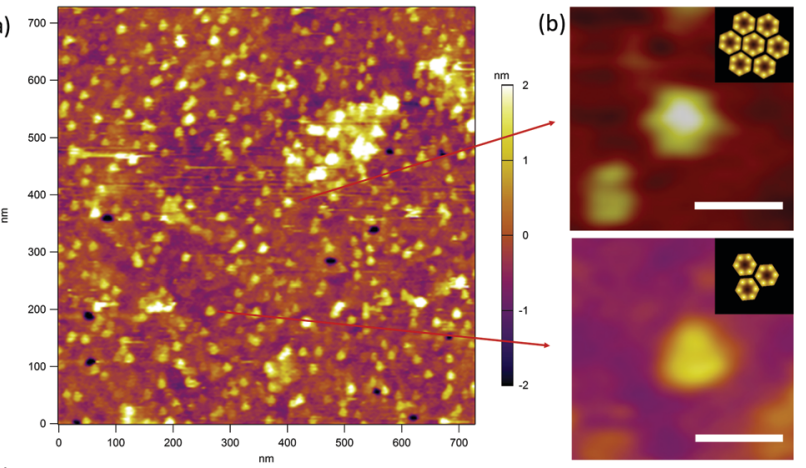

(c)

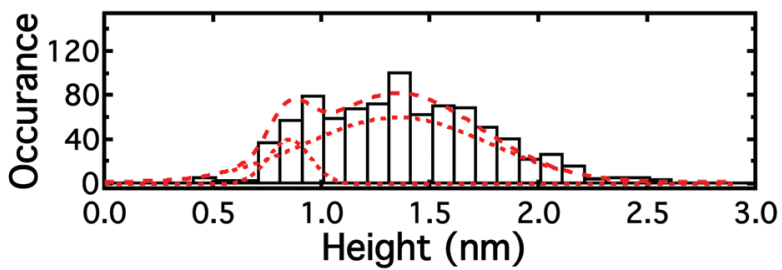

Fig. 4 AFM image of pR-Alexa488 in the DOPC lipid bilayer, at the trap head-region in the longest trap (trap 6, Fig. 3), after the application of an E-field of 180 min. (a) Topology; (b) formation of regular oligomer clusters hexagonal $(n>105)$, triangular $(n>80)$ structures; insets: schematics show the clustering state of pR oligomer (to the scale) ${ }^{38}$ (c) histogram of center height of particles in the AFM image. (Scale bar is $20 \mathrm{~nm}$ in (b)). can be achieved. It should be noted that with AFM we will likely only be "seeing" the immobile or low mobility proteins. This suggests that the mobility of pRs in the trap head-region is greatly reduced after concentration, which may be due to crowding or to oligomer/cluster formation of pRs. ${ }^{40}$ Many of the dots in AFM image appear triangular $(n>80)$ or hexagonal $(n>105)$, Fig. $4 \mathrm{~b}$, and might indicate the occurrence of clusters of pR oligomers. This emergence of clustering, at high protein concentration, may be indicative that the proteins retain their structural form and are not adversely perturbed during the in-plane electrophoresis. The electric field experienced by the pRs during our experiments $\left(\sim 66 \mathrm{~V} \mathrm{~cm}^{-1}\right)$ is several orders of magnitude below those typically experienced in their native environment $\left(\sim 10^{5} \mathrm{~V} \mathrm{~cm}^{-1}\right)$. The height data of particles suggests two populations of pRs that protrude on average by $\sim 0.9$ and $1.4 \mathrm{~nm}$ (Fig. 4c). These would be consistent with two orientations of $\mathrm{pR}$ as mentioned above. Following the electrophoretic concentration in the trap region we find that the ratio between these populations changes from $(1: 1)$ (before E-field) to the $(1: 9)$ (after E-field), with the proteins presenting higher protrusions being the dominant population (89\%). This suggests that pRs with different orientations have different mobility and that the pRs with higher protrusions move more readily in SLBs. Therefore, our approach could also be potentially very useful for the separation of the membrane proteins with different orientations. This is important as, so far, little has been achieved in the controlling the orientations of membrane proteins during or after reconstitution process. ${ }^{41,42}$

In conclusion, this work demonstrates that integral membrane proteins, such as proteorhodopsin $\left(M_{\mathrm{w}} \sim 29 \mathrm{kDa}\right)$ with multiple trans-membrane spanning domains can not only be incorporated into SLBs but that they are able to diffuse freely. Further, under the application of an electric field these proteins move via electrophoresis and by patterning the SLBs into rectangular corrals of different lengths we have been able to demonstrate the controlled increase in protein concentration, following the application of the electric field. The protein concentrations were increased by over seven times, as determined by fluorescence and AFM. The approach is scalable and should permit significantly higher concentrations to be achieved by increasing the size of the trap region thereby opening the possibility of $2 \mathrm{D}$ crystallisation of membrane proteins within such bilayers. It also suggests that electrophoretic separation of integral membrane proteins, whilst still in their membranous environment should be feasible, at least for some membrane proteins. Furthermore, the change in the populations of the two different heights of pRs suggests our method could be used to separate membrane proteins according their orientation post SLB formation.

The authors would like to acknowledge the EPSRC (EP/ I012060/1), the Wellcome Trust ISSF, and NIHR-HTC for Colorectal Therapies for financial support. We also acknowledge Dr George Heath and Dr Johannes R. Roth for helpful discussions.

\section{References}

1 J. P. Overington, B. Al-Lazikani and A. L. Hopkins, Nat. Rev. Drug Discovery, 2006, 5, 993-996. 
2 T. H. Bayburt and S. G. Sligar, FEBS Lett., 2010, 584, 1721-1727.

3 H. Bayley, B. Cronin, A. Heron, M. A. Holden, W. Hwang, R. Syeda, J. Thompson and M. Wallace, Mol. BioSyst., 2008, 4, 1191-1208.

4 C. Yoshina-Ishii and S. G. Boxer, J. Am. Chem. Soc., 2003, 125, 3696-3697.

5 M. Stelzle, R. Miehlich and E. Sackmann, Biophys. J., 1992, 63, 1346-1354.

6 W. Knoll, C. W. Frank, C. Heibel, R. Naumann, A. Offenhäusser, J. Rühe, E. K. Schmidt, W. W. Shen and A. Sinner, Rev. Mol. Biotechnol., 2000, 74, 137-158.

7 L. K. Tamm and H. M. McConnell, Biophys. J., 1985, 47, 105.

8 A. Sumino, T. Dewa, M. Kondo, T. Mori, H. Hashimoto, A. T. Gardiner, R. J. Cogdell and M. Nango, Langmuir, 2011, 27, 1092.

9 H. Sato and J. B. Feix, Biochim. Biophys. Acta, Biomembr., 2006, 1758, $1245-1256$.

10 S. D. Connell, G. Heath, P. D. Olmsted and A. Kisil, Faraday Discuss., 2013, 161, 91-111.

11 Z. V. Leonenko, a. Carnini and D. T. Cramb, Biochim. Biophys. Acta, 2000, 1509, 131-147.

12 L. Wang, J. S. Roth, X. Han and S. D. Evans, Small, 2015, 11, 3306-3318.

13 H. Pace, L. Simonsson Nyström, A. Gunnarsson, E. Eck, C. Monson, S. Geschwindner, A. Snijder and F. Höök, Anal. Chem., 2015, 87, 9194-9203.

14 C. E. Dodd, B. R. G. Johnson, L. J. C. Jeuken, T. D. H. Bugg, R. J. Bushby and S. D. Evans, Biointerphases, 2008, 3, FA59-FA67.

15 P. Jönsson, A. Gunnarsson and F. Höök, Anal. Chem., 2011, 83, 604-611.

16 A. van Oudenaarden and S. G. Boxer, Science, 1999, 285, 1046-1048.

17 M. R. Cheetham, J. P. Bramble, D. G. G. McMillan, L. Krzeminski, X. Han, B. R. G. Johnson, R. J. Bushby, P. D. Olmsted, L. J. C. Jeuken, S. J. Marritt, J. N. Butt and S. D. Evans, J. Am. Chem. Soc., 2011, 133, 6521-6524.

18 X. Han, M. R. Cheetham, K. Sheikh, P. D. Olmsted, R. J. Bushby and S. D. Evans, Integr. Biol., 2009, 1, 205.

19 H. P. Pace, S. D. Sherrod, C. F. Monson, D. H. Russell and P. S. Cremer, Anal. Chem., 2013, 85, 6047-6052.

20 T. Motegi, H. Nabika and K. Murakoshi, Langmuir, 2012, 28, 6656.

21 P. Jonsson, J. McColl, R. W. Clarke, V. P. Ostanin, B. Jonsson and D. Kleneman, Proc. Natl. Acad. Sci. U. S. A., 2012, 109, 10328.
22 M. F. Poyton and P. S. Cremer, Anal. Chem., 2013, 85, 10803-10811.

23 B. Johansson, T. Olsson, P. Jonsson and F. Hook, Soft Matter, 2013, 9, 9414-9419.

24 J. T. Groves and S. G. Boxer, Biophys. J., 1995, 69, 1972-1975.

25 M. Tanaka, J. Hermann, I. Haase, M. Fischer and S. G. Boxer, Langmuir, 2007, 23, 5638-5644.

26 D. J. Olson, J. M. Johnson, P. D. Patel, E. S. G. Shaqfeh, S. G. Boxer and G. G. Fuller, Langmuir, 2001, 17, 7396-7401.

27 J. van Weerd, S. O. Krabbenborg, J. Eijkel, M. Karperien, J. Huskens and P. Jonkheijm, J. Am. Chem. Soc., 2014, 136, 100-103.

28 P. Bertoncello, D. Nicolini, C. Paternolli, V. Bavastrello and C. Nicolini, IEEE Trans. Nanobiosci., 2003, 2, 124.

29 R. B. Jensen, B. R. Kelemen, J. C. McAuliffer and W. C. Smith, US Pat., vol. 20040223323 A1, 2004.

30 C. Brauchle, N. Hampp and D. Oesterhelt, Adv. Mater., 1991, 3, 420.

31 M. J. Hope, M. B. Bally, G. Webb and P. R. Cullis, Biochim. Biophys. Acta, Biomembr., 1985, 812, 55-65.

32 S. Türkcan, M. U. Richly, A. Alexandrou and J.-B. Masson, PLoS One, 2013, 8, e53073.

33 F. Harb, J. Sarkis, N. Ferte and B. Tinland, Eur. Phys. J. E: Soft Matter Biol. Phys., 2012, 35, 1-9.

34 G. Wilhelmina de Groot, S. Demarche, M. G. Santonicola, L. Tiefenauer and G. J. Vancso, Nanoscale, 2014, 6, 2228-2237.

35 L. K. T. Michael and L. Wagner, Biophys. J., 2000, 79, 1400.

36 E. A. Smith, J. W. Coym, S. M. Cowell, T. Tokimoto, V. J. Hruby, H. I. Yamamura and M. J. Wirth, Langmuir, 2005, 21, 9644-9650.

37 S. J. Attwood, Y. Choi and Z. Leonenko, Int. J. Mol. Sci., 2013, 14, 3514-3539.

38 A. L. Klyszejko, S. Shastri, S. A. Mari, H. Grubmüller, D. J. Muller and C. Glaubitz, J. Mol. Biol., 2008, 376, 35-41.

39 S. Hussain, M. Kinnebrew, N. S. Schonenbach, E. Aye and S. Han, J. Mol. Biol., 2015, 427, 1278-1290.

40 S. J. Bussell, D. L. Koch and D. A. Hammer, Biophys. J., 1995, 68, 1836-1849.

41 H. Liang, G. Whited, C. Nguyen and G. D. Stucky, Proc. Natl. Acad. Sci. U. S. A., 2007, 104, 8212-8217.

42 R. Tunuguntla, M. Bangar, K. Kim, P. Stroeve, Caroline M. Ajo-Franklin and A. Noy, Biophys. J., 2013, 105, 1388-1396. 